Relations industrielles

Industrial Relations

\title{
Rodrigue Blouin (sous la direction de), Vingt-cinq ans de pratique en relations industrielles au Québec
}

\section{Jean Boivin}

Volume 46, numéro 2, 1991

URI : https://id.erudit.org/iderudit/050684ar

DOI : https://doi.org/10.7202/050684ar

Aller au sommaire du numéro

Éditeur(s)

Département des relations industrielles de l'Université Laval

ISSN

0034-379X (imprimé)

1703-8138 (numérique)

Découvrir la revue

Citer ce compte rendu

Boivin, J. (1991). Compte rendu de [Rodrigue Blouin (sous la direction de), Vingt-cinq ans de pratique en relations industrielles au Québec]. Relations industrielles / Industrial Relations, 46(2), 482-484.

https://doi.org/10.7202/050684ar

Tous droits réservés (C) Département des relations industrielles de l'Université Laval, 1991
Ce document est protégé par la loi sur le droit d'auteur. L'utilisation des services d'Érudit (y compris la reproduction) est assujettie à sa politique d'utilisation que vous pouvez consulter en ligne.

https://apropos.erudit.org/fr/usagers/politique-dutilisation/ 
l'auteur se livre à une analyse critique des principales théories sociologiques postulant l'existence de systèmes sociaux générateurs de régulation globale. Le concept de statut social permet de classer les groupes sociaux selon une échelle de prestige et d'honneur mais Reynaud estime que la société actuelle est caractérisée par une pluralité des critères d'excellence difficilement compatible avec la thèse fonctionnaliste d'une hiérarchie sociale fondée sur des valeurs culturelles. L'analyse marxiste des rapports de production permet de définir les classes sociales mais l'action ouvrière s'exprime à travers une multitude de conflits catégoriels et localisés réflétant l'effritement de la conscience de classe. Reynaud considère par ailleurs que la vision néomarxiste de Touraine d'une lutte de classe débordant la sphère de la production pour englober tous les aspects de la vie sociale ne rend compte qu'imparfaitement de l'hétérogénéité et de l'autonomie des conflits sociaux. Le principal défaut des raisonnements fonctionalistes et marxistes serait selon Reynaud de supposer l'unité du système social, d'assimiler à une régulation globale l'équilibre instable résultant des interactions des différents systèmes sociaux.

Reynaud voit dans le déclin de l'État, et de l'action collective de masse, non pas la fin des idéologies proclamée par Aron et Bell mais plutôt un recul des régulations de contrôle au profit de systèmes de régulation plus modestes et décentralisés. Les valeurs, les croyances et l'action collective ne sont pas mortes, elles s'expriment sous des formes plus fragmentées que par le passé. L'auteur conclut son ouvrage en se demandant si une science des règles est possible. Les modèles des sciences physiques sont peu utiles en cette matière puisque la régulation sociale, fondée sur l'action collective et le compromis, ne se prête guère à la prévision. Dans les sciences humaines, la prévision tient davantage du conseil que de l'anticipation puisqu'elle influence les décisions des acteurs et peut orienter le cours de l'action; l'étude des règles relève pour l'essentiel du domaine d'une science des aides à la décision.

Cette présentation sommaire de l'ouvrage de Reynaud ne rend pas justice à la richesse des analyses de l'auteur. Elle se veut davantage une invitation à la lecture qu'un exposé rigoureux des thèses défendues par l'un des meilleurs représentants de l'école française des relations industrielles. Cet ouvrage ambitieux, voire même érudit, au cheminement parfois difficile pour le lecteur non averti, ne peut certes pas être considéré comme un manuel d'introduction aux relations industrielles. Il s'adresse plutôt à ceux et celles qui reconnaissent la contribution essentielle de la sociologie à cette entreprise multidisciplinaire qu'est l'étude des relations industrielles.

Reynald Bourque

Université du Québec à Hull

Vingt-cinq ans de pratique en relations industrielles au Québec, sous la direction de Rodrigue Blouin, Les Éditions Yvon Blais inc., Cowansville, Qué., 1990, 1164 p.,

ISBN 2-89073-715-2

Ce colossal ouvrage de plus de 1100 pages a été réalisé à l'occasion du vingt-cinquième anniversaire de la Corporation des conseillers en relations industrielles du Québec. Cet organisme professionnel a voulu ainsi trouver une façon originale et concrète de participer à l'histoire et à l'évolution des relations industrielles au Québec. $\mathrm{M}^{\mathrm{e}}$ Rodrigue Blouin, professeur au Département des relations industrielles et membre actif de la corporation fut chargé d'assumer la responsabilité de l'édition de l'ouvrage qui, l'on s'en doutera, est un collectif d'auteur-e-s et non l'oeuvre d'une seule personne.

Le volume comporte quatre parties. La première s'intitule: «La pratique en relations industrielles» et comprend neuf textes qui touchent les domaines suivants: les orientations en 
relations industrielles; développement organisationnel et relations du travail; la profession de conseiller en relations industrielles (quatre textes); et les organisations patronale et syndicale (trois textes). Les trois autres parties correspondent à ce que le coordonnateur de l'ouvrage appelle dans l'introduction, «les cinq sous-champs de la pratique en relations industrielles, à savoir: les relations du travail, la gestion des ressources humaines et les fonctions conseil, recherche et planification» (p. 2).

On retrouve dix-sept textes sur les relations du travail, dont onze portent sur le processus des rapports collectifs (accréditation de l'association de salariés, monopole de représentation, évolution de la négociation collective, conciliation, médiation, grève et lock-out, dispositions anti-briseurs de grève, services essentiels, convention collective, arbitrage des griefs); on y retrouve également deux textes à portée générale, l'un sur l'évolution du régime de relations du travail et l'autre sur l'évolution du syndicalisme; deux textes sur les régimes de décrets de conventions collectives, le régime général et celui de l'industrie de la construction; et enfin, deux textes sur les rapports individuels de travail dont l'un expose les relations complexes entre le droit civil et la Loi sur les normes du travail en matière de congédiement et l'autre fait état d'une recherche empirique portant sur l'efficacité des recours sur le même sujet en vertu de l'article 124 de la Loi sur les normes du travail.

La troisième partie, consacrée à la fonction gestion des ressources humaines, comprend douze textes dont deux à caractère historique, quatre sur les stratégies de ressources humaines et les nouveaux modes de gestion qui se sont développés au cours des dernières années (qualité totale, cercles de qualité, etc.) et six sur les techniques et modalités de gestion (dotation, plan de carrière, réaffection, condition physique et mode de vie des employés, programme d'aide, personnes vieillissantes).

Enfin, la dernière partie, intitulée «les fonctions conseil, recherche et planification» comprend deux textes sur la discrimination au travail, trois sur le fonctionnement du marché du travail, deux sur les régimes de travail particuliers que sont les normes minimales et la santé et la sécurité du travail et enfin, le dernier texte du volume écrit par le coordonnateur lui-même qui s'intitule «Démocratiser les lieux de travail» et qui constitue une appréciation de l'impact des changements environnementaux sur la législation du travail et de la nécessité, selon l'auteur, de repenser celle-ci afin de tenir compte de tels changements.

La plus grande qualité de cet ouvrage est de réunir sous une même couverture un vaste éventail de textes qui représentent à peu près toutes les facettes des relations industrielles. C'est là un exploit peu commun et digne de mention lorsque l'on connaît la très grande diversité de cette discipline. Il s'agit sans doute d'ailleurs d'une première dans ce domaine pour un ouvrage écrit en langue française.

Cependant, et c'est sans doute inévitable lorsqu'un volume implique la participation d'une cinquantaine d'auteur-e-s, les textes ne sont pas tous de qualité égale. Certains sont très recherchés et poussent l'analyse à un niveau très élevé tandis que d'autres constituent des descriptions anodines de faits et d'expériences diverses. Ce fait est d'ailleurs reconnu par le coordonnateur dans son introduction générale et l'on ne pourrait le tenir responsable de cette situation puisqu'en plus d'avoir à produire un ouvrage à caractère analytique, il devait aussi tenter de faire refléter la réalité du vécu quotidien.

La seule critique de fond que l'on pourrait adresser à ce volume est le choix de l'expression «fonction conseil, recherche et planification» pour décrire l'un des sous-champs des relations industrielles et d'y placer, dans la quatrième partie, des textes portant sur le marché du travail, la discrimination au travail et la santé et la sécurité du travail. On peut difficilement accepter 
cette classification pour deux raisons. D'abord, la fonction «conseil, recherche et planification» ne constitue pas un sous-champ des relations industrielles au même titre que les relations du travail et la gestion des ressources humaines. Cette fonction est le propre de toute discipline ayant une application pratique comme le droit, le service social, la psychologie, etc. D'ailleurs, la fonction «conseil, recherche et planification» s'applique autant au domaine des relations du travail qu'à celui de la gestion des ressources humaines; elle ne peut donc pas être considérée un domaine en soi. Ensuite, elle ne correspond pas du tout aux phénomènes qui sont décrits dans la quatrième partie du volume, à savoir: la discrimination au travail, le marché du travail, et les normes minimales. Ces phénomènes sont du ressort des politiques publiques en matière de travail et il aurait été beaucoup plus simple d'utiliser ce terme pour désigner cette dernière partie. Peut-être le coordonnateur s'est-il senti obligé d'utiliser une expression à consonnance «pragmatique» étant donné la portée souhaitée pour l'ouvrage et les caractéristiques du maître d'oeuvre, c'est-à-dire, la corporation professionnelle? Il n'en reste pas moins que le choix du terme est très malheureux.

Malgré cette dernière critique et le fait que certains textes soient un peu plus faibles que d'autres, Vingt-cinq ans de pratique en relations industrielles au Québec demeure un excellent ouvrage susceptible d'être utile autant aux praticien-ne-s qu'aux étudiant-e-s qui veulent se familiariser avec le champ d'étude des relations industrielles.

Jean BoIvin

Université Laval

Unions and Communities Under Siege: American Communities and the Crisis of Organized

Labor, by Gordon L. ClARK, England, Cambridge University Press, 1989, 309 p., ISBN 0-521-36516-3.

Gordon Clark echoes Tip O'Neil's "All politics is local politics" with his thesis that it is "in terms of the local context of labor-management relations" that the "current crisis of organized labor ought to be considered." While there is a large and growing literature of community-based studies of unions, workers and labor relations, these are primarily the work of historians. It is as a Professor of Geography that Clark adds a spatial dimension to the analysis expressed in the book's title, Unions and Communities Under Siege: American Communities and the Crisis of Organized Labor. Published in 1989 as part of Cambridge University Press' series on Human Geography, the dedication to his grandfather, "a railway worker and unionist of another time and place" indicates the author's sympathies.

Clark suggests that workers and unions have ultimately been more victim than beneficiary of the fact that, "[f]ostering decentralization has been an integral goal of federal labor law." Other commentators (e.g. George Brooks) have decried the existence of multi-local and multiemployer negotiations because they believe these large scale bargaining structures undermine the Wagner Act's guarantee to workers of "unions of their own choosing." Clark, on the other hand, says that the purpose of organizing is negotiating and effective negotiating requires a union structure matching the employers organization and power. He therefore concludes that worksite-based bargaining units fragment national (and international) union power. He contends:

There can be no doubt that the Wagner Act (1935) and the Taft-Hartley (1947) amendments made it relatively more difficult to organize labor in the United States than in other advanced countries. Unions have had to organize firms on a plant-by-plant basis, seeking representation rights for workers in different communities across the country. 